\title{
Modified Newton method to determine multiple zeros of nonlinear equations
}

\author{
RAJINDER THUKRAL
}

\author{
Padé Research Centre, 39 Deanswood Hill, Leeds, West Yorkshire, LS17 5JS, England
}

\begin{abstract}
A new one-point iterative method for solving nonlinear equations is constructed. It is proved that the new method has a convergence order of three. Per iteration the new method requires two evaluations of the function. Kung and Traub conjectured that the multipoint iteration methods, without memory based on $n$ evaluations, could achieve maximum convergence order $2^{n-1}$ but the new method produces convergence order of three, which is better than the expected maximum convergence order of two. Hence, we demonstrate that the conjecture fails for a particular set of nonlinear equations. Numerical comparisons are included to demonstrate the exceptional convergence speed of the proposed method using only a few function evaluations.
\end{abstract}

Keywords: Modified Newton method; Nonlinear equations; Kung-Traub's conjecture; Maximum order of convergence; Efficiency index; One-point method.

Subject Classifications: AMS (MOS): 65H05.

\section{INTRODUCTION}

In this paper, we present a new one-point third-order iterative method to find multiple roots of the nonlinear equation $f(x)=0$, where $f: I \subset \mathfrak{R} \rightarrow \mathfrak{R}$ for an open interval where I is a scalar function. The root-solver is of great practical importance in Science and engineering. In recent years many modifications of the Newton-type methods for simple roots have been proposed and analysed [3] and little work has been done on multiple roots. In this paper, we are interested in the case that $\alpha$ is a root of multiplicity $m>1$ of a nonlinear equation, that is $f^{k}(\alpha)=0$, $k=0,1,2 \ldots m-1$ and $f^{m}(\alpha) \neq 0$. Hence the prime motive of this study is to develop a new class of iterative method for finding multiple roots of nonlinear equations of a higher order than the classical Newton method [3,6,11]. The purpose of this paper is to show further development of the one-point third-order method [10]. This paper is actually a continuation of the previous study [10]. The extension of this investigation is based on the one-point third-order method for finding multiple roots of nonlinear equations. In addition, the new iterative method has a better precision than the Dong method, the Thukral method or the Neta et al. methods [1,2,5,8]. Hence the proposed one-point third-order method is significantly better when compared with these established methods.

The classical modified Newton method for finding multiple roots is given by

$x_{n+1}=x_{n}-m \frac{f\left(x_{n}\right)}{f^{\prime}\left(x_{n}\right)}$,

which converges quadratically [3,6,7,11]. For the purpose of this paper, we improve the classical modified Newton method and construct a new one-point third-order iterative method for finding multiple roots of nonlinear equations. The one-point third-order method presented in this paper only uses two evaluations of the function per iteration. Kung and Traub conjectured that the multipoint iteration methods, without memory based on $\mathrm{n}$ evaluations, could achieve optimal convergence order $2^{n-1}$. In fact, we have obtained a higher order of convergence than the maximum order of convergence suggested by the Kung and Traub conjecture [4]. We demonstrate that the Kung and Traub conjecture fails for a particular case that is when the multiple root of a nonlinear equation is equal to zero.

The remaining sections of the paper are organised as follows: some basic definitions relevant to the present work are stated in section 2. In section 3, the new one-point third-order iterative method is defined and proved. In section 4, five well-established two-point third-order methods are stated and we will demonstrate the effectiveness of the new one-point third-order iterative method. Finally, in section 5, numerical comparisons are made to demonstrate the performance of the new one-point third-order method.

\section{BASIC DEFINITIONS}

In order to establish the order of convergence of the iterative methods, some of the definitions are stated: 
Definition 1 Let $f(x)$ be a real function with a simple root $\alpha$ and let $\left\{x_{n}\right\}$ be a sequence of real numbers that converge towards $\alpha$. The order of convergence $\mathrm{p}$ is given by

$\lim _{n \rightarrow \infty} \frac{x_{n+1}-\alpha}{\left(x_{n}-\alpha\right)^{p}}=\lambda \neq 0$

where $p \in \mathfrak{R}^{+}$and $\lambda$ is the asymptotic error constant, [3,6,7,11].

Definition 2 Let $e_{k}=x_{k}-\alpha$ be the error in the kth iteration, then the relation

$e_{k+1}=\zeta e_{k}^{p}+\mathrm{O}\left(e_{k}^{p+1}\right)$

is the error equation. If the error equation exists then $p$ is the order of convergence of the iterative method, [3,6,7,11].

Definition 3 Let $r$ be the number of function evaluations of the iterative method. The efficiency of the iterative method is measured by the concept of efficiency index and defined as

$\sqrt[r]{p}$

where $\mathrm{p}$ is the order of convergence of the method, [7].

Definition 4 (Kung and Traub conjecture) Let $x_{n+1}=g\left(x_{n}\right)$ define an iterative function without memory with kevaluations. Then

$$
p(g) \leq p_{\text {opt }}=2^{k-1}
$$

where $p_{o p t}$ is the maximum order [4].

Definition 5 suppose that $x_{n-1}, x_{n}$ and $x_{n+1}$ are three successive iterations closer to the root $\alpha$ of (1). Then the computational order of convergence may be approximated by

$\mathrm{COC} \approx \frac{\ln \left|\delta_{n} \div \delta_{n-1}\right|}{\ln \left|\delta_{n-1} \div \delta_{n-2}\right|}$

(6)

where $\delta_{i}=f\left(x_{i}\right) \div f^{\prime}\left(x_{i}\right)$, [9].

\section{CONSTRUCTION OF THE NEW METHOD AND ANALYSIS OF CONVERGENCE}

In this section we define a new one-point third-order method for finding multiple roots of a nonlinear equation. In fact the new iterative method is an improvement of the classical modified Newton method, given by (1). The one-point third-order Newton method is expressed by

$x_{n+1}=x_{n}-m \frac{f\left(x_{n}\right)}{f^{\prime}\left(x_{n}\right)}-\left(2 x_{n}\right)^{-1}\left[x_{n}^{2}-\left(m \frac{f\left(x_{n}\right)}{f^{\prime}\left(x_{n}\right)}\right)^{2}\right]$,

where $x_{0}$ is the initial guess and provided that denominators of (7) are not equal to zero. Now we shall verify the convergence property of the new one-point third-order iterative method (7).

Theorem 1 Let $\alpha \in I$ be a multiple root of multiplicity m of a sufficiently differentiable function $\quad f: I \subset \mathfrak{R} \rightarrow \mathfrak{R}$ for an open interval I. If the initial guess $x_{0}$ is sufficiently close to $\alpha$ then the convergence order of the new method defined by (7) is three.

Proof Let $\alpha$ be a multiple root of multiplicity $m$ of a sufficiently smooth function $f(x), e=x-\alpha$.

Using the Taylor expansion of $f(x)$ about $\alpha$, we have 
$f\left(x_{n}\right)=\left(\frac{f^{(m)}(\alpha)}{m !}\right) e_{n}^{m}\left[1+c_{1} e_{n}+c_{2} e_{n}^{2}+c_{3} e_{n}^{3}+\cdots\right]$.

$f^{\prime}\left(x_{n}\right)=\left(\frac{f^{(m)}(\alpha)}{(m-1) !}\right) e_{n}^{m-1}\left[1+\left(\frac{m+1}{m}\right) c_{1} e_{n}+\left(\frac{m+2}{m}\right) c_{2} e_{n}^{2}+\cdots\right]$.

where

$c_{k}=\frac{m ! f^{(m+k)}(\alpha)}{(m+k-1) ! f^{(m)}(\alpha)}$.

Dividing (8) by (9), we have

$\frac{f\left(x_{n}\right)}{f^{\prime}\left(x_{n}\right)}=\frac{e_{n}}{m}-\frac{c_{2} e_{n}^{2}}{m^{2}}+\frac{\left[(m+1) c_{2}^{2}-2 m c_{3}\right] e_{n}^{3}}{m^{3}}+\cdots$.

and

$\left(\frac{f\left(x_{n}\right)}{f^{\prime}\left(x_{n}\right)}\right)^{2}=\frac{e_{n}^{2}}{m^{2}}-\frac{2 c_{2} e_{n}^{3}}{m^{3}}+\frac{\left[(2 m+3) c_{2}^{2}-4 m c_{3}\right] e_{n}^{4}}{m^{4}}+\cdots$.

Substituting (11) and (12) in (7), we obtain

$e_{n+1}=e_{n}-m \frac{f\left(x_{n}\right)}{f^{\prime}\left(x_{n}\right)}-\left(2 e_{n}\right)^{-1}\left[e_{n}^{2}-\left(m \frac{f\left(x_{n}\right)}{f^{\prime}\left(x_{n}\right)}\right)^{2}\right]$,

Simplifying (13), yields

$e_{n+1}=\frac{c_{2}^{2} e_{n}^{3}}{2 m^{2}}$.

This reveals that the one-point iterative method (7) reaches third order of convergence by using only two functional evaluations per iteration. This completes the proof.

\section{THE ESTABLISHED METHODS}

For the purpose of comparison, five two-point third-order methods presented in $[1,2,5,8,12]$ are considered. Since these methods are well established, we state the essential formulas used to calculate the simple root of nonlinear equations and thus compare the effectiveness of the new one-point third-order method.

\subsection{The Thukral two-point third order method}

Thukral [8] developed the third-order Newton-type method, given by

$y_{n}=x_{n}-\frac{f\left(x_{n}\right)}{f^{\prime}\left(x_{n}\right)}$,

$x_{n+1}=x_{n}-\frac{(m-1)^{(m-1)} f\left(x_{n}\right)^{2}}{(m-1)^{(m-1)} f^{\prime}\left(x_{n}\right) f\left(x_{n}\right)-m^{(m-1)} f^{\prime}\left(x_{n}\right) f\left(y_{n}\right)}$.

\subsection{The Dong two-point third order method}

Dong [1] presented a third-order Newton-type method, given by

$y_{n}=x_{n}-\sqrt{m} \frac{f\left(x_{n}\right)}{f^{\prime}\left(x_{n}\right)}$, 
$x_{n+1}=y_{n}-m\left(1-\frac{1}{\sqrt{m}}\right)^{1-m}\left(\frac{f\left(y_{n}\right)}{f^{\prime}\left(x_{n}\right)}\right)$.

Second of Dong's two-point third-order method [2] is given by

$$
\begin{aligned}
& y_{n}=x_{n}-\left(\frac{m}{m+1}\right)\left(\frac{f\left(x_{n}\right)}{f^{\prime}\left(x_{n}\right)}\right), \\
& x_{n+1}=y_{n}-\left(\frac{m}{m+1}\right)\left[\frac{f\left(x_{n}\right)}{\left(1+m^{-1}\right)^{m} f^{\prime}\left(y_{n}\right)-f^{\prime}\left(x_{n}\right)}\right] .
\end{aligned}
$$

\subsection{The Neta two-point third order method}

Neta [5] developed a third-order variant of the Newton-type method, given by

$$
\begin{aligned}
& y_{n}=x_{n}-\left(\frac{m}{2}\right)\left(\frac{m+3}{m+1}\right)\left(\frac{f\left(x_{n}\right)}{f^{\prime}\left(x_{n}\right)}\right), \\
& x_{n+1}=x_{n}-\left(\beta_{1}+\beta_{2}\left(\frac{f\left(y_{n}\right)}{f\left(x_{n}\right)}\right)\right)\left(\frac{f\left(x_{n}\right)}{f^{\prime}\left(x_{n}\right)}\right), \\
& \text { where } \beta_{1}=\frac{m^{3}+4 m^{2}+9 m+2}{(m+3)^{2}} \text { and } \beta_{2}=\frac{2^{m+1}\left(m^{2}-1\right)}{(m+3)^{2}[(m-1) /(m+1)]^{m}} .
\end{aligned}
$$

\section{APPLICATION OF THE NEW ONE-POINT THIRD-ORDER METHOD}

The present one-point third-order method given by (7) is employed to solve nonlinear equations with multiple roots. To demonstrate the performance of the new one-point third-order method, four particular nonlinear equations are used. The consistency and stability of results is determined by examining the convergence of the new iterative method. The findings are generalised by illustrating the effectiveness of the new method for determining the multiple roots of a nonlinear equation. Consequently, estimates are given of the approximate solutions produced by the methods considered and a list of errors obtained by each of the methods. The numerical computations listed in the tables were performed on an algebraic system called Maple. In fact, the errors displayed are of absolute value and insignificant approximations by the various methods have been omitted in the following tables.

The new one-point third-order method requires two function evaluations and has the order of convergence three. To determine the efficiency index of the new method, definition 3 will be used. Hence, the efficiency index of the new iterative method given by $(7)$ is $\sqrt[2]{3} \approx 1.7132$. and the efficiency index of the two-point third-order methods considered in this 
paper is given by (16), (18), (20), (22), (25) is $\sqrt[3]{3} \approx 1.4423$. It is shown that the efficiency index of the new one-point third-order method is much better than the other similar methods. The difference between the multiple roots $\alpha$ and the approximation $x_{n}$ for test functions with initial guess $x_{0}$ are displayed in tables. In fact, $x_{n}$ is calculated by using the same total number of function evaluations for all methods. Furthermore, the computational order of convergence approximations (COC) are displayed in tables. From the tables we observe that the COC perfectly coincides with the theoretical result. However, this is the case when initial guess are reasonably close to the sought zeros.

\subsection{Numerical example 1}

In our first example we will demonstrate the convergence of the new one-point third-order Newton-type method for the following nonlinear equation

$$
f(x)=\left[e^{x} \sin (x)+\ln \left(1+x^{2}\right)\right]^{8},
$$

having multiplicity $m=8$ and the exact value of the multiple roots of (27) is $\alpha=0$. In Table 1 are the errors obtained by each of the Newton-type methods described, based on the initial value $x_{0}=2^{-1}$. We observe that the new one-point thirdorder Newton-type method is converging by the order three.

Table 1 Errors occurring in the estimates of the root of (27) by the methods described

\begin{tabular}{ccccccc}
\hline methods & $\left|x_{1}-\alpha\right|$ & $\left|x_{2}-\alpha\right|$ & $\left|x_{3}-\alpha\right|$ & $\left|x_{4}-\alpha\right|$ & $\mid f\left(x_{4}\right)$ & $C O C$ \\
$(1)$ & $0.639 \mathrm{e}-1$ & $0.660 \mathrm{e}-2$ & $0.850 \mathrm{e}-04$ & $0.144 \mathrm{e}-07$ & $0.189 \mathrm{e}-62$ & 2.0004 \\
$(16)$ & $0.195 \mathrm{e}-1$ & $0.234 \mathrm{e}-4$ & $0.440 \mathrm{e}-13$ & $0.296 \mathrm{e}-39$ & $0.581 \mathrm{e}-316$ & 3.0000 \\
$(18)$ & $0.235 \mathrm{e}-1$ & $0.572 \mathrm{e}-4$ & $0.949 \mathrm{e}-12$ & $0.434 \mathrm{e}-35$ & $0.126 \mathrm{e}-282$ & 3.0000 \\
$(22)$ & $0.490 \mathrm{e}-2$ & $0.690 \mathrm{e}-6$ & $0.183 \mathrm{e}-17$ & $0.344 \mathrm{e}-52$ & $0.196 \mathrm{e}-419$ & 2.9726 \\
$(20)$ & $0.172 \mathrm{e}-1$ & $0.128 \mathrm{e}-4$ & $0.563 \mathrm{e}-14$ & $0.476 \mathrm{e}-42$ & $0.265 \mathrm{e}-338$ & 3.0000 \\
$(25)$ & $0.203 \mathrm{e}-1$ & $0.287 \mathrm{e}-4$ & $0.892 \mathrm{e}-13$ & $0.268 \mathrm{e}-38$ & $0.269 \mathrm{e}-308$ & 3.0000 \\
$(7)$ & $0.816 \mathrm{e}-2$ & $0.102 \mathrm{e}-5$ & $0.214 \mathrm{e}-17$ & $0.197 \mathrm{e}-52$ & $0.226 \mathrm{e}-421$ & 3.0000 \\
\hline
\end{tabular}

\subsection{Numerical example 2}

In our second example we will demonstrate the convergence of new Newton-type iterative methods for a different type of nonlinear equation

$$
f(x)=\left[e^{-x}-\cos (x)\right]^{3}
$$

having multiplicity $m=3$ and the exact value of the multiple roots of (28) is $\alpha=0$. In Table 2 are the errors obtained by each of the methods described, based on the initial value $x_{0}=2^{-3}$. In this particular case we observe that all the Newton-type methods are performing better than expected.

Table 2 Errors occurring in the estimates of the root of (28) by the methods described

\begin{tabular}{cclllll}
\hline methods & $\left|x_{1}-\alpha\right|$ & $\left|x_{2}-\alpha\right|$ & $\left|x_{3}-\alpha\right|$ & $\left|x_{4}-\alpha\right|$ & $\left|f\left(x_{4}\right)\right|$ & $C O C$ \\
$(1)$ & $0.198 \mathrm{e}-1$ & $0.378 \mathrm{e}-3$ & $0.143 \mathrm{e}-6$ & $0.204 \mathrm{e}-13$ & $0.846 \mathrm{e}-41$ & 2.0000 \\
$(16)$ & $0.182 \mathrm{e}-2$ & $0.440 \mathrm{e}-8$ & $0.616 \mathrm{e}-25$ & $0.169 \mathrm{e}-75$ & $0.479 \mathrm{e}-227$ & 3.0000 \\
$(18)$ & $0.296 \mathrm{e}-2$ & $0.297 \mathrm{e}-7$ & $0.300 \mathrm{e}-22$ & $0.309 \mathrm{e}-67$ & $0.295 \mathrm{e}-202$ & 3.0000 \\
$(22)$ & $0.270 \mathrm{e}-2$ & $0.550 \mathrm{e}-8$ & $0.486 \mathrm{e}-25$ & $0.334 \mathrm{e}-76$ & $0.373 \mathrm{e}-229$ & 2.9873 \\
$(20)$ & $0.655 \mathrm{e}-3$ & $0.968 \mathrm{e}-10$ & $0.312 \mathrm{e}-30$ & $0.104 \mathrm{e}-91$ & $0.114 \mathrm{e}-275$ & 3.0000 \\
$(25)$ & $0.250 \mathrm{e}-2$ & $0.147 \mathrm{e}-7$ & $0.297 \mathrm{e}-23$ & $0.247 \mathrm{e}-70$ & $0.151 \mathrm{e}-211$ & 3.0000 \\
$(7)$ & $0.156 \mathrm{e}-2$ & $0.191 \mathrm{e}-8$ & $0.350 \mathrm{e}-26$ & $0.215 \mathrm{e}-79$ & $0.995 \mathrm{e}-239$ & 3.0000 \\
\hline
\end{tabular}




\subsection{Numerical example 3}

In this subsection we take another nonlinear equation. We will demonstrate the convergence of the new Newton iterative methods for the following nonlinear equation

$$
f(x)=\left[(x-1)^{6}-1\right]^{100},
$$

having multiplicity $m=100$ and the exact value of the multiple roots of (29) is $\alpha=0$. In Table 3 are the errors obtained by each of the methods described, based on the initial value $x_{0}=-3^{-1}$. Here also, we observe that all the Newton-type methods are converging by the expected order.

Table 3 Errors occurring in the estimates of the root of (29) by the methods described

\begin{tabular}{ccccccc}
\hline methods & $\left|x_{1}-\alpha\right|$ & $\left|x_{2}-\alpha\right|$ & $\left|x_{3}-\alpha\right|$ & $\left|x_{4}-\alpha\right|$ & $\left|f\left(x_{4}\right)\right|$ & $C O C$ \\
$(1)$ & 0.151 & $0.415 \mathrm{e}-1$ & $0.392 \mathrm{e}-2$ & $0.381 \mathrm{e}-4$ & $0.856 \mathrm{e}-364$ & 2.0374 \\
$(16)$ & $0.555 \mathrm{e}-1$ & $0.456 \mathrm{e}-3$ & $0.276 \mathrm{e}-9$ & $0.616 \mathrm{e}-28$ & $0.611 \mathrm{e}-2743$ & 3.0002 \\
$(18)$ & $0.763 \mathrm{e}-1$ & $0.207 \mathrm{e}-2$ & $0.533 \mathrm{e}-7$ & $0.921 \mathrm{e}-21$ & $0.166 \mathrm{e}-2025$ & 3.0008 \\
$(22)$ & - & - & - & - & - & - \\
$(20)$ & $0.548 \mathrm{e}-1$ & $0.427 \mathrm{e}-3$ & $0.221 \mathrm{e}-9$ & $0.303 \mathrm{e}-28$ & $0.107 \mathrm{e}-2773$ & 3.0002 \\
$(25)$ & $0.558 \mathrm{e}-1$ & $0.469 \mathrm{e}-3$ & $0.304 \mathrm{e}-9$ & $0.835 \mathrm{e}-28$ & $0.933 \mathrm{e}-2730$ & 3.0002 \\
$(7)$ & $0.340 \mathrm{e}-1$ & $0.106 \mathrm{e}-3$ & $0.369 \mathrm{e}-11$ & $0.157 \mathrm{e}-33$ & $0.211 \mathrm{e}-3302$ & 3.0000 \\
\hline
\end{tabular}

\subsection{Numerical example 4}

In the final example, we take another type of nonlinear equation. We will demonstrate the convergence of the new Newton iterative method for the following nonlinear equation

$$
f(x)=\left[\ln (1+x)+x^{3}\right]^{25}
$$

having multiplicity $m=25$ and the exact value of the multiple roots of (30) is $\alpha=0$. In Table 4 are the errors obtained by each of the methods described, based on the initial value $x_{0}=5^{-1}$. Here, we also find that all the Newton-type methods are converging by the expected order.

Table 4 Errors occurring in the estimates of the root of $(30)$ by the methods described

\begin{tabular}{ccccccc}
\hline methods & $\left|x_{1}-\alpha\right|$ & $\left|x_{2}-\alpha\right|$ & $\left|x_{3}-\alpha\right|$ & $\left|x_{4}-\alpha\right|$ & $\left|f\left(x_{4}\right)\right|$ & $C O C$ \\
$(1)$ & $0.362 \mathrm{e}-3$ & $0.654 \mathrm{e}-7$ & $0.214 \mathrm{e}-14$ & $0.229 \mathrm{e}-29$ & $0.986 \mathrm{e}-741$ & 2.0000 \\
$(16)$ & $0.104 \mathrm{e}-1$ & $0.112 \mathrm{e}-5$ & $0.145 \mathrm{e}-17$ & $0.318 \mathrm{e}-53$ & $0.355 \mathrm{e}-1337$ & 2.9740 \\
$(18)$ & $0.843 \mathrm{e}-2$ & $0.409 \mathrm{e}-6$ & $0.492 \mathrm{e}-19$ & $0.853 \mathrm{e}-58$ & $0.187 \mathrm{e}-1451$ & 2.9779 \\
$(22)$ & $0.592 \mathrm{e}-2$ & $0.437 \mathrm{e}-6$ & $0.167 \mathrm{e}-18$ & $0.924 \mathrm{e}-56$ & $0.138 \mathrm{e}-1400$ & 2.9761 \\
$(20)$ & $0.100 \mathrm{e}-1$ & $0.985 \mathrm{e}-6$ & $0.962 \mathrm{e}-18$ & $0.896 \mathrm{e}-54$ & $0.639 \mathrm{e}-1351$ & 2.9745 \\
$(25)$ & $0.103 \mathrm{e}-1$ & $0.111 \mathrm{e}-5$ & $0.140 \mathrm{e}-17$ & $0.280 \mathrm{e}-53$ & $0.147 \mathrm{e}-1338$ & 2.9740 \\
$(7)$ & $0.328 \mathrm{e}-6$ & $0.440 \mathrm{e}-20$ & $0.106 \mathrm{e}-61$ & $0.150 \mathrm{e}-186$ & $0.233 \mathrm{e}-4670$ & 3.0000 \\
\hline
\end{tabular}




\section{CONCLUSIONS}

In this paper, the performance of the new one-point third-order Newton-type method has been demonstrated. The prime motive for presenting the new iterative method was to improve the classical modified Newton method. The effectiveness of the new one-point third-order method has been examined by showing the accuracy of the multiple root of a nonlinear equation. The main purpose of demonstrating the new Newton-type method for several types of nonlinear equations was purely to illustrate the accuracy of the approximate solution, the stability of the convergence, the consistency of the results and to determine the efficiency of the new iterative method. It has been shown numerically and verified that the new Newton-type methods converge by the order three. Empirically, we have found that the new one-point third-order method is very effective when the multiple roots are zeros. The main advantages are: very high computational efficiency; the new method is not limited to the Kung and Traub conjecture; a better efficiency index; a one-point one-step iteration method; very competitive with the two-point third-order methods. Finally, further investigation is required to overcome the drawback of the new one-point method namely that is it is only effective when the multiple roots are zeros.

\section{REFERENCES}

[1] C. Dong, A basic theorem of constructing an iterative formula of higher order for computing multiple roots of equation, Math. Numer. Sinica 11 (1982) 445-450.

[2] C. Dong, A family of multipoint iterative functions for finding multiple roots of equations, Inter. J. Comp. Math. 21 (1987) 363-367.

[3] W. Gautschi, Numerical Analysis: an Introduction, Birkhauser, 1997.

[4] H. Kung, J. F. Traub, Optimal order of one-point and multipoint iteration, J. Assoc. Comput. Math. 21 (1974) 643651.

[5] B. Neta, New third order nonlinear solvers for multiple roots, Appl. Math. Comput. 202 (2008) 162-170.

[6] M. S. Petkovic, B. Neta, L. D. Petkovic, J. Dzunic, Multipoint methods for solving nonlinear equations, Elsevier 2013.

[7] A. M. Ostrowski, Solutions of equations and system of equations, Academic Press, New York, 1960.

[8] R. Thukral, A new third-order iterative method for solving nonlinear equations with multiple roots, J. Math. Comput. 6 (2010) 61-68.

[9] R. Thukral, New modifications of Newton-type methods with eighth-order convergence for solving nonlinear equations, J. Adv. Math. Vol 10 (3) (2015) 3362-3373.

[10] R. Thukral, New modification of Newton method with third-order convergence for solving nonlinear equations of type $f(0)=0$, Amer. J. Comput. Appl. Math. 6 (1) (2016).

[11] J. F. Traub, Iterative Methods for solution of equations, Chelsea publishing company, New York 1977.

[12] H. D. Victory, B. Neta, A higher order for multiple zeros of nonlinear functions, Inter. J. Comp. Math. 12 (1983) 329-335. 